\title{
Everolimus verlängert progressionsfreies Überleben bei Pankreastumoren
}

Der mTOR-Inhibitor Everolimus kann das progressionsfreie Überleben von Patienten mit fortgeschrittenen neuroendokrinen Pankreastumoren mehr als verdoppeln - bei einer niedrigen Rate an schweren Nebenwirkungen. Dies zeigten James C. Yao und Kollegen vom M.D. Anderson Cancer Center in Houston, TX/USA in einer aktuell im New England Journal of Medicine veröffentlichten Studie [Yao JC et al. N Engl J Med 2011; 364:514-23]. In der prospektiven Phase-III-Studie behandelten sie insgesamt 410 Patienten mit fortgeschrittenen neuroendokrinen Pankreastumoren. Alle Patienten hatte in den vorangegangenen 12 Monate eine Progression erlitten. Randomisiert erhielten sie entweder Everolimus in einer Dosierung von $10 \mathrm{mg}$ p.o. täglich $(n=207)$ oder ein Placebo $(n=203)$. Darüber hinaus bekamen alle Patienten eine adäquate Supportivtherapie (best supportive care). Das mediane progressionsfreie Überleben betrug unter der Everolimus-Therapie elf Monate; unter Placebo waren es nur 4,6 Monate (Hazard Ratio für Krankheitsprogression oder Tod im Everolimus-Arm: 0,35; $95 \%-K I$ 0,27-0,45; $\mathrm{p}<0,001)$. Wie zu erwarten wur- den therapiebedingte Nebenwirkungen vor allem im Everolimus-Arm beobachtet. In der Mehrzahl waren es Nebenwirkungen ersten und zweiten Grades. Die im New England Journal of Medicine veröffentlichte Studie wurde von Novartis-Oncology finanziert. Everolimus inhibiert mTOR (mammalian target of rapamycin), ein Protein, das eine wichtige Rolle in der Regulation des Zellzyklus einnimmt. Seine Inhibition führt zur Arretierung der Zelle in der G-Phase des Zellzyklus. Dies wiederum kann zur Reduktion des Tumorwachstums führen. Primär wurde Everolimus eingeführt, um Abstoßungsreaktionen bei Organtransplantationen zu verhindern. Mittlerweile wird es auch in der Tumortherapie eingesetzt. Seit 2009 ist es zur Therapie von fortgeschrittenen Nierenzellkarzinomen zugelassen.

\section{Carina Jung}

Yao JC, Shah MH, Ito T et al. Everolimus for Advanced Pancreatic Neuroendocrine Tumors. N Engl J Med 2011; 364:514-23, Pressemitteilung des M.D. Anderson Cancer Center, University of Texas vom 9.2.2011: Everolimus improves progression-free survival for patients with rare pancreatic cancer

\section{Sinkender Trend}

\section{Für 2011 weniger Krebstote in Europa erwartet als 2007}

Die Mortalitätsraten durch Krebs sinken voraussichtlich im Jahr 2011 - Epidemiologen erwarten, dass weniger Männer an Lungen- und kolorektalen Tumoren sterben und weniger Frauen an Brustkrebs. Wissenschaftler aus Italien und der Schweiz haben ein für die USA entwickeltes System zur Ermittlung der Todesursachenstatistik erstmals für Europa angewendet. Die Arbeitsgruppe um die Epidemiologen Matteo Malvezzi und Carlo La Vecchia vom Mario-Negri-Institut in Mailand nahmen als Basis Daten aus den 27 EU-Mitgliedstaaten (Stand 2007) von 1970 bis 2007. Sie berechneten daran die Todesraten für jedes Jahr und identifizierten Trends. Anhand dieser Trends schätzten sie die Entwicklung für $2011 \mathrm{ab}$. Für dieses Jahr erwarten Malvezzi und Kollegen EU-weit 1.281.436 Krebstote. Bei den Männern sind dies mit standardisierten Raten von 142,8 pro 100.000 Einwohner insgesamt $7 \%$ weniger als 2007 (153,8/100.000). Die Sterberate durch Tumoren bei Frauen wird voraussichtlich um $6 \%$ sinken (von 90,7/100.000 im Jahr
2007 auf 85,3/100.000 in 2011). „Die geschätzten Raten stimmen überein mit Prognosen für die Krebssterblichkeit in Europa bis 2015, die sinkende Raten und eine annähernd konstante absolute Anzahl an Todesfällen ansetzen“, betonen die Epidemiologen. Trotz der generell positiven Trends nehmen Malvezzi und Kollegen an, dass ein bestehendes Gefälle in der Krebssterblichkeit zwischen den zentral- und osteuropäischen Ländern gegenüber Westeuropa in näherer Zukunft bestehen bleiben wird. Sie führen die insgesamt sinkenden Mortalitätsraten vor allem darauf zurück, dass die Brustkrebstodesrate bei Frauen und die Sterberate durch Lungentumoren und kolorektale Tumoren bei Männern sinken.

\footnotetext{
Carina Jung

Malvezzi M, Arfe A, Bertuccio P et al. European cancer mortality predictions for the year 2011. Ann Oncol. 2011 Feb 8. [Epub ahead of print], doi:10.1093/annonc/mdq774; Pressemitteilung der Oxford University Press vom 7.2.2011: Researchers predict neraly 1.3 million cancer death in Europe in 2011
} 